Int. J. Dev. Biol. 61: 415-425 (2017)

doi: $10.1387 / \mathrm{ijdb} .160399 \mathrm{cy}$

\title{
Apolipoprotein C-I mediates Wnt/Ctnnb1 signaling during neural border formation and is required for neural crest development
}

\author{
CHIKA YOKOTA*,1, CAROLINA ÅSTRAND ${ }^{1}$, SHUJI TAKAHASHI ${ }^{2}$, DANIEL W. HAGEY ${ }^{1}$ and JAN M. STENMAN ${ }^{1}$ \\ ${ }^{1}$ Ludwig Institute for Cancer Research Ltd, Stockholm, Sweden and \\ 2 Institute for Amphibian Biology, Graduate School of Science, Hiroshima University, Japan
}

\begin{abstract}
In vertebrates, the neural crest and placodes originate in the neural border, which is located between the neural plate and epidermal ectoderm. The neural crest and placodes give rise to a vast array of cell types. Formation of neural crest is a multi-step process, in which Wnt signals are used reiteratively, but it is currently not clear if a Wnt signal is required for neural border formation. Here, we have identified apolipoprotein $C$-I (apoc1) in a screen for genes regulated by Wnt/ Ctnnb1 signaling in late blastula stage Xenopus tropicalis embryos. We show that Xenopus laevis apoc1 encodes a small, secreted protein, and is induced by Wnt/Ctnnb1 signaling. Depletion of Apoc1 protein results in a neural border formation defect and loss of border fates, including neural crest cells. However, unlike anotherWnt/Ctnnb1 target, $g b \times 2.2$, apoc1 is not required for patterning of the neural border. We further show that $g b \times 2.2$ and apoc1 are independently regulated by Wnt signaling. Our results thus suggest that Wnt regulates border formation and patterning by distinct genetic mechanisms.
\end{abstract}

KEY WORDS: apolipoprotein, Xenopus, neural crest, neural plate border, wnt, ctnnb1

\section{Introduction}

Neural crest cells constitute a transient cell population that is unique to vertebrate embryos. Together with the placodes, this multipotent and self-renewing cell population forms the glia and neurons of the peripheral nervous system. In addition, the neural crest contributes a vast array of other cell types to the developing embryo, such as blood vessels, melanocytes, and craniofacial cartilage and bones (Sauka-Spengler and Bronner-Fraser 2008). Development of neural crest cells has been suggested to occur in five sequential steps: neural border induction, neural crest induction, neural crest specification, neural crest migration and neural crest differentiation (Pegoraro and Monsoro-Burq 2013). In Xenpous, Wnt, Bmp, and Fgf signals begin patterning the ectoderm already during the blastula stage resulting in the formation of neural ectoderm, epidermal ectoderm, and the neural border (hereafter referred to as the border) from which both neural crest and placodal cells originate (Pegoraro and Monsoro-Burq 2013).

Conflicting reports exist on the involvement of Wnt signals in border formation. While one study proposed that an initial Wnt signal is required to initiate Bmp-mediated border formation (Patthey et al., 2009), another suggested that Wnt activity initially need to be repressed for border formation (Steventon and Mayor 2012). Induced border cells are characterized by expression of border specifiers, e.g. members of the $d l x, m s x$, pax and zic families, which stabilize the border fate (Patthey and Gunhaga 2011; Milet and Monsoro-Burq 2012). At gastrula stages, regionalization of the border then allows induction of neural crest cells and placodal cells from the posterior and anterior border, respectively. Later, at neurula stages, the posterior border is further regionalized resulting in formation of neural crest from the medial border and posterior placodes from the lateral border. Wnt is required for posteriorizing the border, and gbx2.2 mediates this activity (Patthey et al., 2008;

\footnotetext{
Abbreviations used in this paper: Apoc1, apolipoprotein C-I; BMP, bone morphogenetic protein; Chrd, chordin; Ctnnbl, catenin betal; Fgf, fibroblast growth factor; Gbx2.2, gastrulation brain homeobox2, gene2; MO, morpholino oligo; WISH, whole mount in situ hybridization.
}

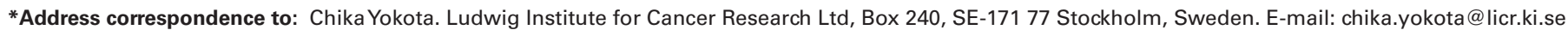
Tel: (+46) 8-52487-866. Fax: (+46) 8-34-1960. Web: http://www.ludwigcancerresearch.org/location/stockholm-branch - iD http://orcid.org/0000-0002-8380-2556
}

Supplementary Material (four figures and 5 tables) for this paper is available at: http://dx.doi.org/10.1387/ijdb.160399cy

Received: 10 August, 2016. Accepted: 21 October, 2016. Edited by: Makoto Asashima.

ISSN: Online 1696-3547, Print 0214-6282 
Li et al., 2009). Induction of neural crest requires the combined action of the border specifiers and signals, including Wnt, Bmp, and Fgf, produced by surrounding tissues (Hong et al., 2008). For example, pax3 and zic1 are capable of inducing neural crest in the ventral Xenopus embryo, but only if Wnt signaling is not blocked (Sato et al., 2005). Neural crest induction results in the activation of neural crest specifiers, including snai2, foxd3, and sox9, which initiate epithelial-to-mesenchymal transition, neural crest cell migration and differentiation (Duband 2010; Milet and Monsoro-Burq 2012). The timing of differentiation is correlated with the final fate/ destination of the specific cell type (Theveneau and Mayor 2012).

Members of the Wnt family are secreted glycoproteins, which can activate at least three intracellular signaling pathways. The most intensively studied pathway is the Wnt/Ctnnb1 pathway (formerly "canonical pathway") that regulates cellular processes by Ctnnb1-mediated transcriptional activation of downstream target genes. In Ctnnb1-depleted embryos, the neural plate is dramatically expanded and border fates suppressed, suggesting that $c t n n b 1$ is required to inhibit neural induction and to promote the specification of border fates, possibly by regulating neural border

\section{A}

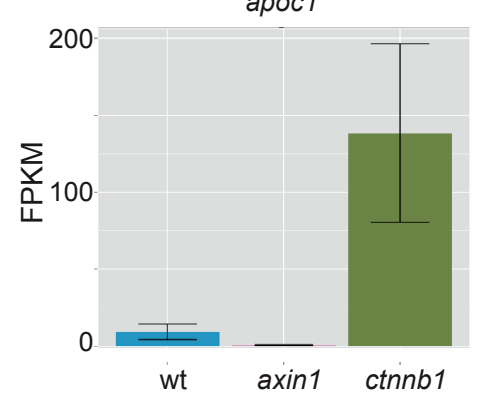

D

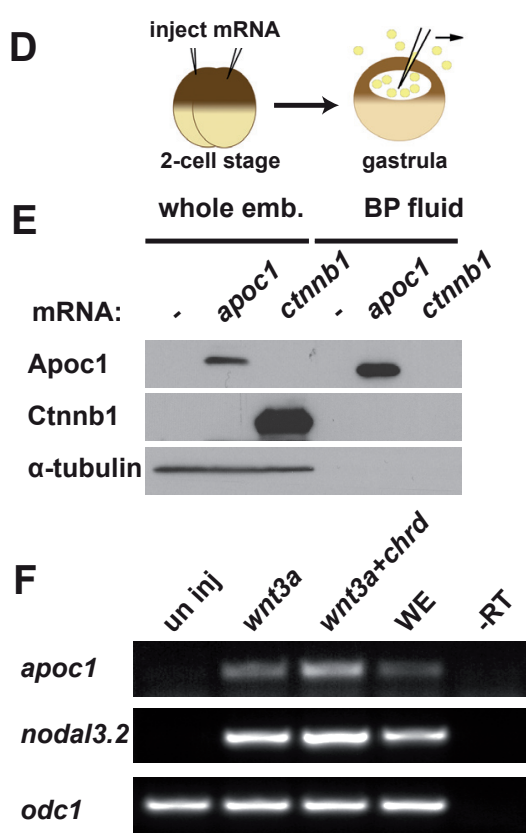

E

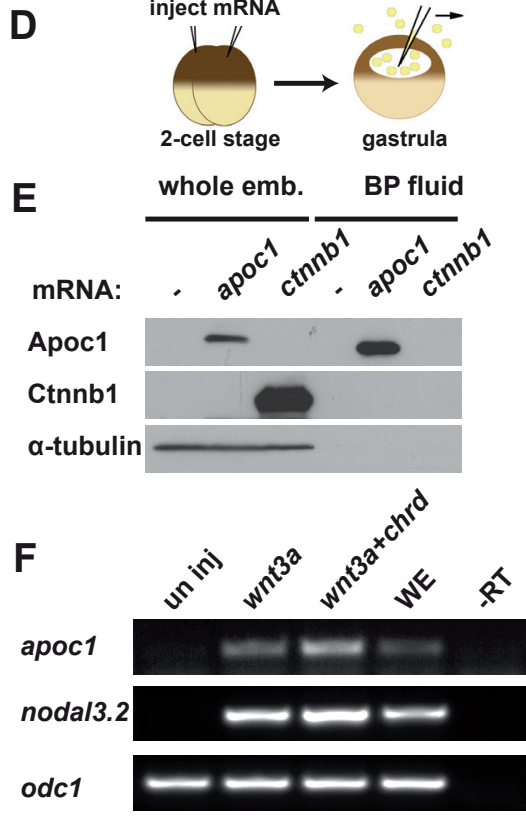

mRNA:
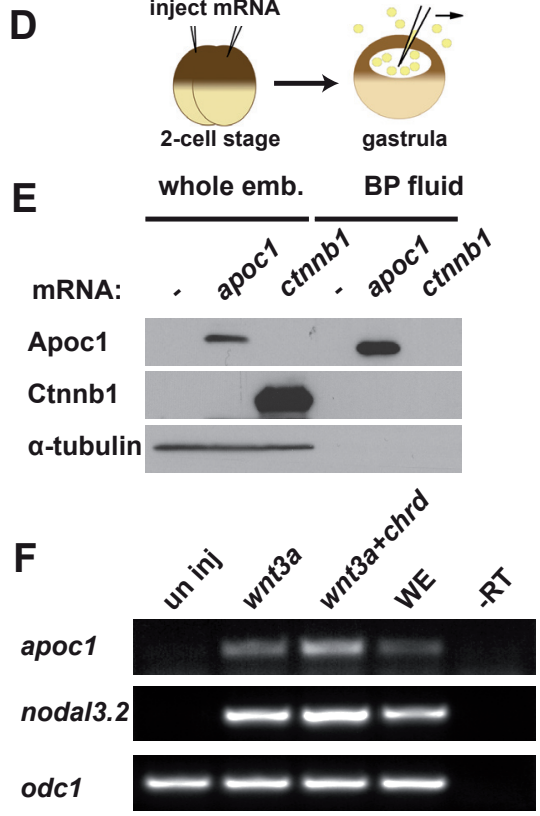

B

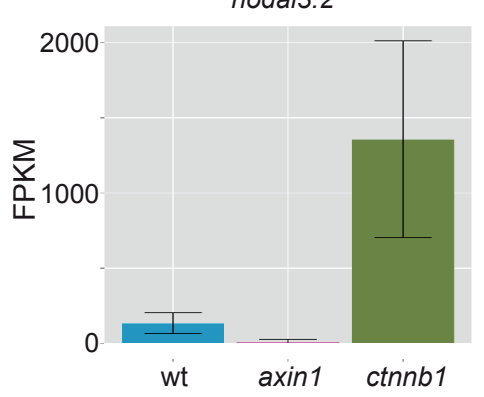

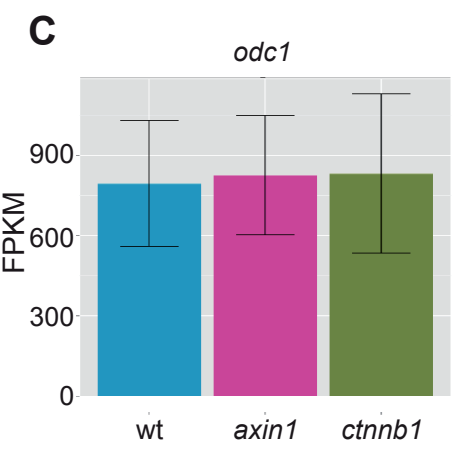

\section{G cycloheximide \\ DEX}

apoc1
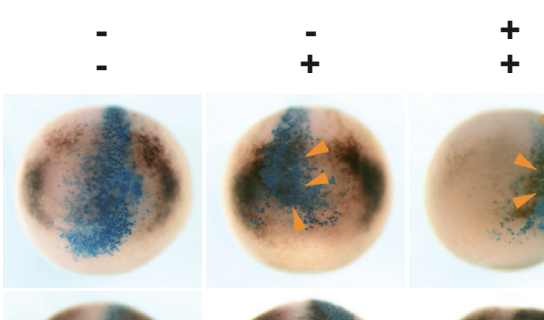

Fig. 1. Identification, characterization and regulation of Xenopus apoc1. (A-C) Bar charts showing the expression levels, displayed as fragments per kilobase of transcript per million fragments mapped (FPKM), for apoc1 (A), the Wnt regulated gene nodal3.2 (B), and the non-Wnt regulated gene odc1 (C) in Xenopus tropicalis embryos that were either uninjected (blue), injected with 105 pg axin1 mRNA (pink) or 24 pg <N-HA-ctnnb1 mRNA (green). (D) Schematic drawing of the Apoc1 secretion experiment. mRNA was injected at the two-cell stage. The blastopore fluid was collected at the gastrula stage and subjected to western blot (WB) analysis to determine whether flag-tagged Apoc1 protein is secreted from cells during embryonic development. (E) Apoc1, but not Ctnnb1, protein was detected by WB in the blastocoel fluid after injection of flag-tagged apoc1 (500 pg) and flag-tagged ctnnb1 (1 ng). The tubulin blot shows that there is no cellular contamination in the blastopore fluid. (F) Wnt regulation of apoc1 in Xenopus laevis embryos. (F) $300 \mathrm{pg}$ of wnt3a or $200 \mathrm{pg}$ of chrd and $300 \mathrm{pg}$ wnt3a were injected into the animal side of two blastomeres at the two-cell stage. Animal cap explants were dissected from stage 9 embryos, cultured until stage 10, and processed for RT-PCR analysis. Expression of apoc1, and the known Wnt target gene nodal3.2, was induced by wnt3a alone, and chrd+wnt3a. Odc1 was used as loading control. (G) Apoc1, gbx2.2 and pax3 are immediate-early target gene of Wnt/Ctnnb1 signaling. 200 pg of GR-ctnnb1 and $100 \mathrm{pg}$ lacZ mRNA were injected into one side of a dorsoanimal blastomere of 8-cell stage embryos and the embryos were treated with cycloheximide 30 min prior to DEX treatment at stage 11.5. The expression of apoc1, gbx2.2, pax3 and snail2 were examined by whole mount in situ hybridization. Orange arrows indicate ectopic localization of the tested genes. 
formation (Heeg-Truesdell and LaBonne 2006). In addition, ectopic expression of ctnnb1 will posteriorize the anterior border (Li et al., 2009). Thus, available data suggests that both Wnt ligands and Ctnnb1 transcriptional activity is instrumental for the developmental program that ultimately results in formation of neural crest cells.

In adult mice and humans, members of the apoliporotein family bind lipids to form lipoprotein particles. They function in lipid transport, but also as coenzymes. In addition, they can interact with lipoprotein receptors (Bolanos-Garcia and Miguel2003). Mouse and human apolipoprotein c1 (apoc1) may influence memory functions and contribute to the pathogenesis of Alzheimer's disease (Berbee et al., 2011). In adult Xenopus laevis, apoc1 is predominantly expressed in the liver, but also in the stomach, gonads, intestines, and ovarian follicles during late oogenesis (Gohin et al., 2010). However, a role for apoc1 during early vertebrate development has not been reported.

In a screen for Wnt/Ctnnb1-regulated genes in late blastula stage Xenopus tropicalis embryos, we identified a strongly regulated transcript, which encodes for apoc1. Using Xenopus laevis, we demonstrate that Wnt can activate apoc 1 in both embryos and ecodermal explants. Furthermore, we show that apoc1 is required for development of border cells fates, including neural crest cells. Previously, gbx2.2 has been suggested to be the earliest Wnt/ Ctnnb1 target in neural crest formation (Li et al., 2009). However, we show that $g b \times 2$.2and apoc 1 are independently regulated by Wnt and mediate separate activities. Our data suggest a model where apoc 1 functions as a critical mediator of Wnt in border formation. However, apoc1 is not required for patterning of the border along the anteroposterior axis, a function performed rather by $g b x 2.2$.

\section{Results}

\section{Identification, characterization, and regulation of Xenopus apoc1}

To better understand the role of Wnt/Ctnnb1 signaling in early development, e.g. in ectoderm patterning, we attempted to discover novel Wnt target genes by comparing the transcriptional profiles of late blastula (Nieuwkoop and Faber stage 9.5) Xenopus tropicalis embryos where Wnt/Ctnnb1 signaling is normal (uninjected embryos), decreased (embryos injected with axin $1 \mathrm{mRNA}$ ) or increased (embryos injected with a constitutively active form of ctnnb1 mRNA; Fig. $1 \mathrm{~A}-\mathrm{C}$ ). We identified an unannotated genomic region, scaffold_701:348314-352838 (JGI v4.1), predicted by TopHat/Cufflinks to encode a transcript that exhibits strong Wnt/Ctnnb1 regulation (uninjected: 9,44 Fragments Per Kilobase of transcript per Million fragments mapped (FPKM), axin1 injected 0,70 FPKM, ctnnb1 injected: 138,33 FPKM; Fig. 1A). Inspection of the genomic region in the UCSC genome browser revealed numerous unspliced ESTs with 3 or 4 exons. A nucleotide BLAST analysis of the genomic region identified a RefSeq predicted mRNA(XM_002941032.1) with four exons, encoding an 85 amino acid protein (XP_002941078) containing an Apolipoprotein C-I conserved domain (pfam04691). Further analysis using BLASTP of the predicted Xenopus tropicalis protein identified the 89 amino acid Xenopus laevis Apolipoprotein C-I (Apoc1) protein (ADD51575.1) as the closest ortholog with 66/89 (74\%) identical and 78/89 (87\%) positive amino acids.

We used SignalP 4.1 to analyze the Xenopus laevis and tropicalis Apoc1 protein sequences for possible signal peptides (www.cbs. $\mathrm{dtu} . d k /$ services/SignalP). Both proteins contain a putative signal
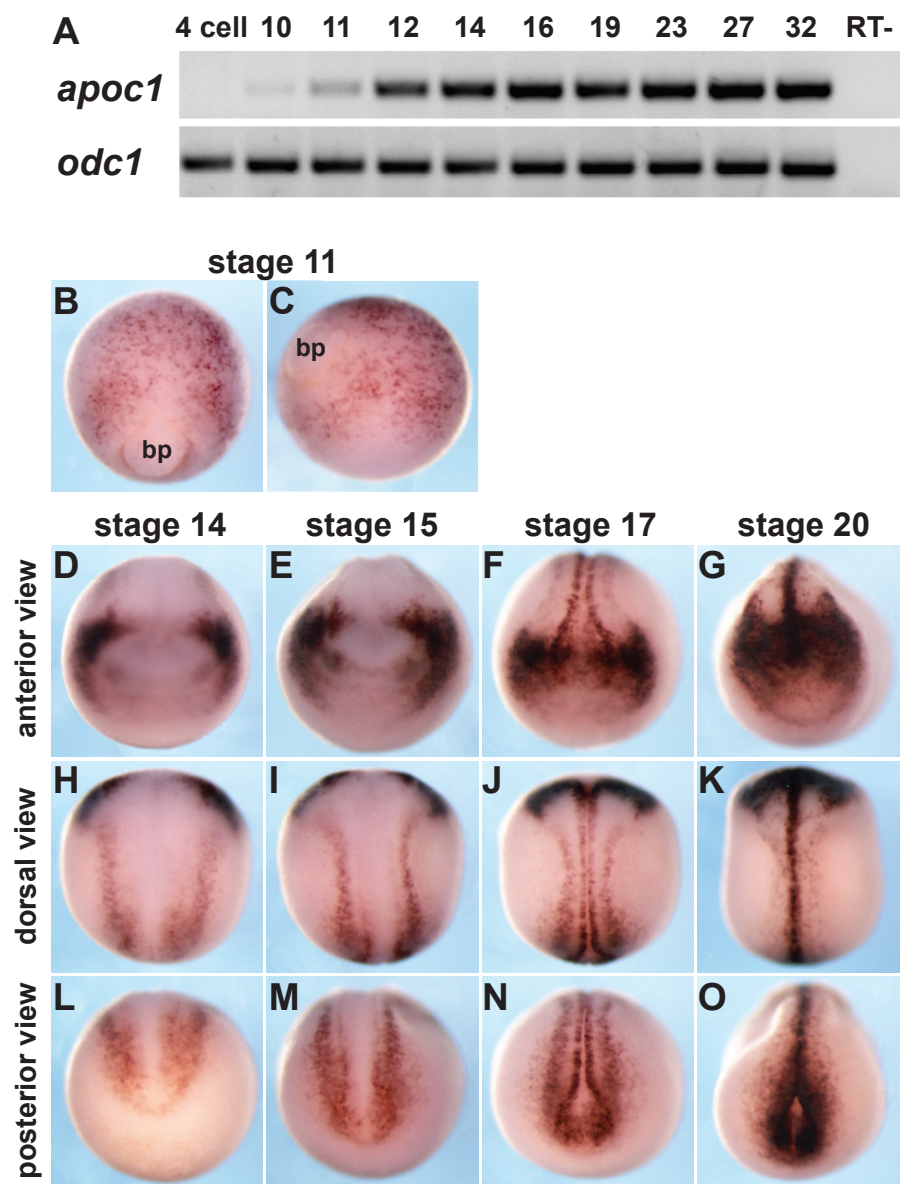


$\mathbf{N}$

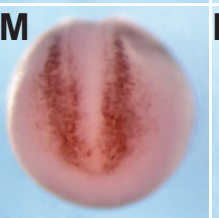

$\mathbf{N}$

0
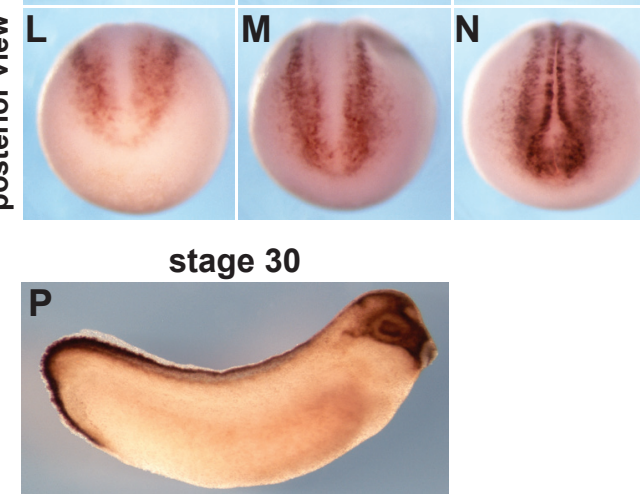

Fig. 2. Spatiotemporal expression pattern of Xenopus laevis apoc1. (A) The temporal expression pattern of apoc1 during Xenopus development (4-cell stage, stages 10-32) was determined by RT-PCR (RT- is without reverse transcriptase from stage 32). (B-P) The spatiotemporal expression pattern of apoc1 determined by whole mount in situ hybridization. (B,C) Apoc1 expression at stage 11; bp - the closing blastopore. Anterior view (D-G), dorsal view (H-K), posterior view (L-O) of stage 14 (D, H, L), stage $15(\mathbf{E}, \mathbf{I}, \mathbf{M})$, stage $17(\mathbf{F}, \mathbf{J}, \mathbf{N})$ and stage $20(\mathbf{G}, \mathbf{K}, \mathbf{O})$ embryos. (P) Lateral view of a stage 30 embryo.

peptide, but no transmembrane domain, suggesting that they are secreted proteins. To test whether Apoc1 is secreted in Xenopus embryos, we injected C-terminally flag-tagged Xenopus laevis apoc1 or flag-tagged ctnnb1 mRNA at the 4-cell stage and collected the blastocoel fluid from stage 10 (early gastrula) embryos (Fig. 1D). As predicted, Apoc1, but not Ctnnb1, was detected by Western blot analysis in the blastocoel fluid (Fig. 1E).

Our screen established that apoc1 is Wnt/Ctnnb1 regulated in Xenopus tropicalis. To further test whether Wnt/Ctnnb1 signaling regulates apoc1 expression also in Xenopus laevis, we injected wnt3a, the BMP antagonist chordin/wnt3a mRNA into embryos 
and performed RT-PCR on dissected animal caps at stage 10. As predicted, both apoc1 and the well-established direct Wnt/Ctnnb1 target gene nodal3.2 were strongly upregulated (Fig. 1F). We then asked if apoc1 is a direct target of Wnt/Ctnnb1 signaling in Xenopus laevis (Fig. 1G). Previous studies have shown that both gbx2.2 and pax3 but not snail2 were direct targets of Wnt/Ctnnb1 signaling (de Croze et al., 2011; Li et al., 2009), and our result in Fig $1 \mathrm{G}$ confirmed this. Strong ectopic expression of apoc1, gbx2.2 and pax3 were observed overlapping area where GR-Ctnnb1 was expressed even in the cycloheximide treated embryos. These data suggested that apoc1 is a direct target of Wnt/Ctnnb1 signaling.

\section{Apoc1 is expressed at the neural plate border and in neural crest cells}

We next determined the temporal expression profile of apoc1 in Xenopus laevis embryos (Fig. 2A). While we could not detect any expression by RT-PCR analysis in 4-cell stage embryos, we found low levels of apoc1 in early gastrula (stage 10) embryos, consistent with our screen data, detecting low-level expression in late blastula (stage 9.5) Xenopus tropicalis embryos. The expression level increased dramatically by late gastrula (stage 12) and remained high during embryonic development (until stage 32; tadpole stage).

To determine the spatiotemporal distribution of apoc1 mRNAs, we performed whole mount in situ hybridization (WISH) on stages 11 to 30 (gastrula to tadpole) Xenopus laevis embryos (Fig. 2 B-P). Robust Apoc1 expression was first detected by WISH at stage

A

Translation blocking MO AGTCTCGAAAGATTTCTTCCACGGG

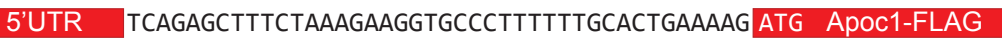
GCCGCCACC ATG Apoc1-FLAG

B

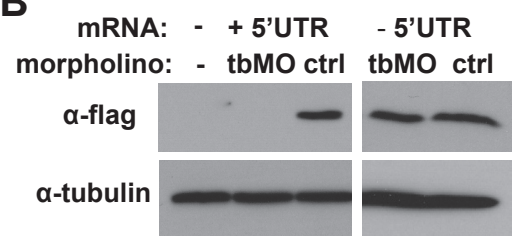

C

apoc1

odc

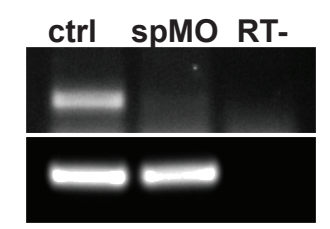

D
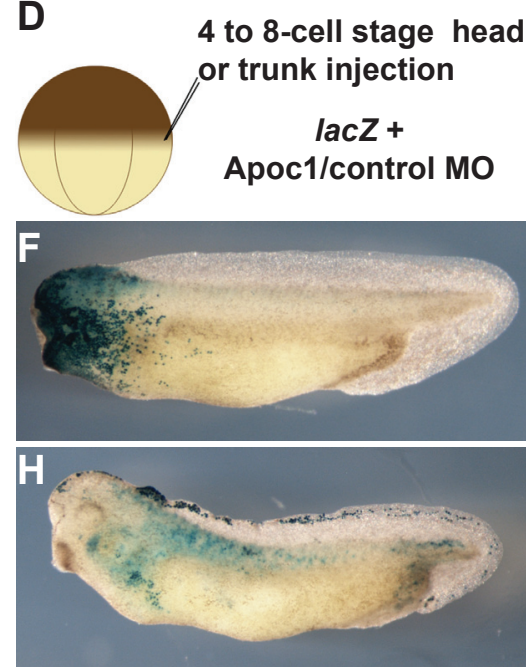

10.5 in the dorsal animal region (data not shown). At stage 11, the area of expression stretched towards the dorso-anterior and dorso-lateral part of the embryo along the gastrulation movement (Fig. 2 B,C). At the neurula stage, apoc1 was highly expressed in parts of the preplacodal ectoderm, cranial neural crest cells, posterior neural plate border cells, the neural folds, and migrating neural crest cells (Fig. 2D-O). In stage 30 embryos, expression remained high in the dorsal fin, which consists of neural crest derived cell types (Fig. 2P). At this stage, apoc1 expression was also detected in cranial placodes, including the lens placode, and in the mandibular neural crest. In summary, the apoc1 expression pattern suggests a function for apoc1 in neural crest and cranial placode development.

\section{Apoc1 is required for neural crest development}

To determine whether apoc 1 is required for neural crest development in vivo, we designed a translation blocking morpholino antisense oligo (tbMO) to prevent Apoc1 protein synthesis (Fig. 3A). As the genome sequence for Xenopus laevis was not yet available, an analysis of more than 50 expressed sequence tags (ESTs) was performed to identify a tbMO binding site that was conserved in the 5 prime untranslated sequence (5'UTR) of all ESTs. To determine the efficiency of the Apoc 1 tbMO, we co-injected it (or control MO) with +/-5'UTR flag-tagged apoc1 mRNA (Fig. 3A) and analyzed the Apoc1 protein expression levels in gastrula stage embryos. The Apoc1 tbMO efficiently blocked translation of apoc1 mRNA only when it contained the tbMO target sequence (Fig. 3B). We also designed a splice blocking $\mathrm{MO}$ (spMO) against apoc1 and assayed its efficiency by RT-PCR (Fig. 3C). Expression of apoc1 mRNA was detected in stage 10 embryos that had been injected with control $\mathrm{MO}$, but not in embryos injected with the spMO suggesting that it efficiently blocked the splicing of apoc1 pre-mRNA (Fig. 3C).

We next analyzed the consequences of Apoc1 depletion in Xenopus embryos by co-injecting the Apoc1 tbMO with lacZ mRNA, encoding for beta-galactosidase, as a lineage tracer (Fig. 3D). The tbMO was first targeted to the presumptive cranial neural crest region from which craniofacial cartilage and bone forms (Fig. 3F, G). We

Fig. 3. Loss of neural crest derivatives in Apoc1 depleted embryos. (A) The Apoc1 translational blocking MO (tbMO) targets the 5' UTR region of the Xenopus laevis apoc1 gene. To determine the efficiency of the tbMO, two flag-tagged apoc1 constructs were used differing only in the region upstream of the ATG start site. (B) Western blot using an anti-flag antibody on lysates from embryos injected with 300 pg 5'UTR-apoc1-flag or apoc1-flag mRNA and $40 \mathrm{ng}$ Apoc1 tbMO or $40 \mathrm{ng}$ control $\mathrm{MO}$ (ctrl). (C) $30 \mathrm{ng}$ of the splice blocking morpholino (spMO) or control MO (ctrl) was injected into the animal side of two blastomeres at the two-cell stage and the embryos were assayed by RT-PCR at stage 10.5. Odc is used as loading control. (D) Schematic drawing of the experiment. $40 \mathrm{ng}$ Apoc $1 \mathrm{MO}$ was injected into either the presumptive head (F,G) or trunk/tail (H,I) region together with $200 \mathrm{pg}$ lacZ mRNA. (E) Embryos injected with $40 \mathrm{ng}$ control MO were normal. $(\mathbf{F}, \mathbf{G})$ Injections targeted to the head region resulted in $76.1 \%(n=134)$ of the embryos displaying small or missing eyes and head deformation. $(\mathbf{H}, \mathbf{I})$ Injections targeted to the trunk region resulted in perturbed dorsal fin development in $55.2 \%(n=38)$ of the embryos. The pictures in $\mathbf{( G , I ) ~ w e r e ~ t a k e n ~ p r i o r ~ t o ~ X - g a l ~ s t a i n i n g ~} \mathbf{( F , H )}$ 
allowed the embryos to develop until they reached the tadpole stage. Apoc1 depleted embryos exhibited clear head developmental defects including smaller or missing eyes $(76.1 \%, n=134$; supplementary material Table S1). Next, we targeted the tbMO to the presumptive trunk neural crest region from which cells of the dorsal fin derives (Fig. $3 \mathrm{H}, \mathrm{I})$. Clear defects in dorsal fin formation were observed (55.3\%, n=38; Fig. $3 \mathrm{H}$, I; supplementary material Table S1), with the heads unperturbed. Control MO injected embryos exhibited no developmental defects (Fig. 3E). To further strengthen these observations, we injected the spMO into the presumptive cranial or trunk neural crest regions, and, as expected, we observed head developmental $(56.4 \%, \mathrm{n}=55)$ or dorsal fin formation defects $(45.2 \%, n=31$ ) respectively (Fig. $S 1$; supplementary material Table $\mathrm{S1}$ ). As both the tbMO and spMO efficiently depleted the Apoc1 protein levels and resulted in identical phenotypes, we mainly used the tbMO in subsequent experiments.

Given that apoc1 expression was detected prior to neural crest induction, we next wanted to determine whether apoc1 is essential for neural crest emergence. Thus, we co-injected Apoc1 tbMO together with lac $Z$ mRNA, and analyzed the expression of the neural crest markers $c-m y c$, sox 9 , snai2 and twist 1 by in situ

hybridization (Fig. 4A). Consistent with an early role for apoc1 in neural crest development, expression of these genes was missing or significantly downregulated on the injected side of early neurula stage embryos (supplementary material Table S2). Furthermore, depletion of apoc1 mRNA with the spMO also resulted in reduced snail2, sox9, twist1, pax3and zic1 expression (Fig. S2; supplementary material Table S2). The reduction/loss of neural crest markers could be rescued by injecting tbMO resistant apoc1 mRNA(Fig. 4B upper panel; supplementary material Table S2), but not by injecting apoc1 mutant mRNA lacking signal sequence (Fig. 4B lower panel; Fig. S3; supplementary material Table S2). These results demonstrate that the morpholino phenotype is indeed caused by a specific depletion of Apoc1 protein, and secretion is required for its function.

Bellmeyer and colleagues have shown that inhibition of Wnt signaling results in lost $c$-myc expression already in gastrula stage embryos, and that $c-m y c$ is required for expression of definitive neural crest markers (Bellmeyer et al., 2003). Apoc1 depletion resulted in reduced or completely lost $c$-myc expression, placing apoc1 downstream of wnt, but upstream of $c-m y c$. Furthermore, $c-m y c$ has been demonstrated to regulate id3 (Light et al., 2005). We therefore examined id3, which controls the

A

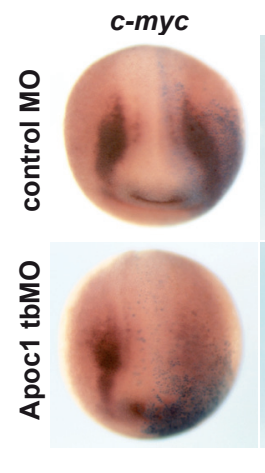

B

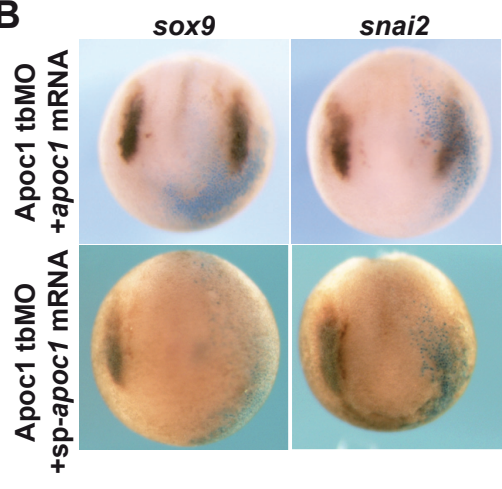

C

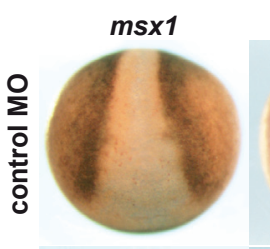

pax3
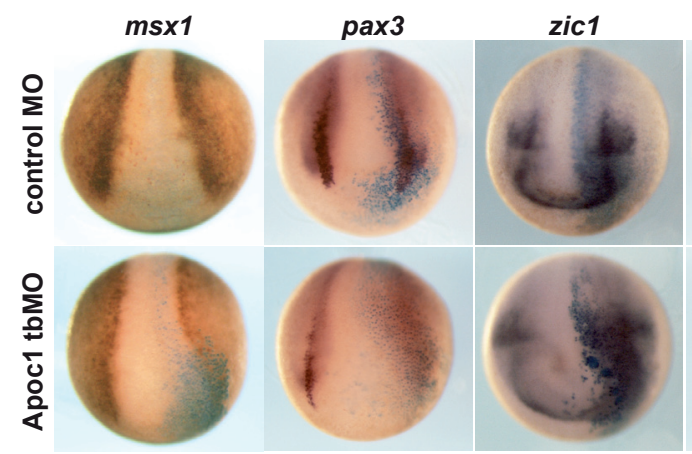

sox9
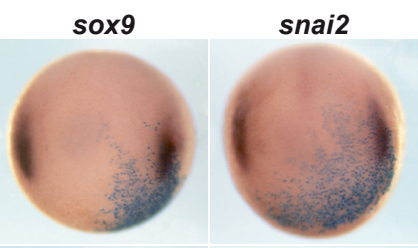

twist1
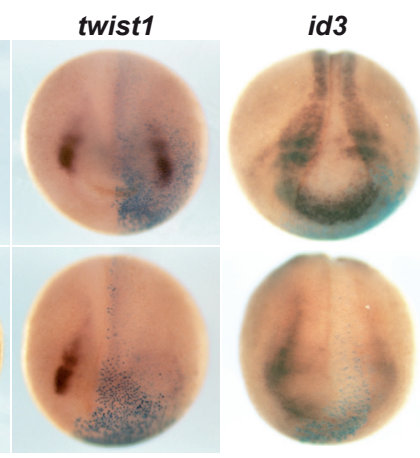
maintenance of neural crest stem cell behavior, inApoc1-depleted embryos. Indeed, id3expression was downregulated or missing (Fig. 4A; supplementary material Table S2).

Neural crest cells originate from the border between the neural and epidermal ectoderm. The expression of neural crest markers is preceded by expression of border specifiers, such as $m s x 1$, pax3 and zic1 (Fig. 4C). C-myc depleted embryos also exhibit border defects, suggesting that $c-m y c$ also can be considered a border specfier. More specifically, in C-myc morphants, zic1 and the neural precursor marker sox2 are both expanded laterally. In Apoc1 depleted embryos, zic1 and sox2 are both expanded laterally (Fig. 4C; supplementary material Table S2), and the medial boundary of $x k 81 a 1$ (keratin), which is expressed in epider-

Fig. 4. Apoc1 is required for neural border formation and neural crest emergence. (A) Whole mount in situ hybridization for the neural crest (NC) markers c-myc, sox9, snai2, twist1, id3. Control MO injected embryos are shown in upper panel and Apoc1 tbMO injected ones are shown in lower panel. (B) The reduction of sox9 and snai2 expression by the Apoc1 tbMO could be rescued by coinjection of $400 \mathrm{pg}$ of apoc1 mRNA (upper panel) but not by coinjection of 400 pg of sp-apoc1 mRNA (lower panel). (C) Whole mount in situ hybridization for the neural plate border specifiers msx1, pax3, zic1, the neural marker sox2, and the non-neural ectoderm markerxk81a1. Control MO injected embryos are shown in upper panel and Apoc1 tbMO injected ones are shown in lower panel. One of the dorsal blastomeres was injected with $30 \mathrm{ng}$ of either control MO (upper panels in A, C) or Apoc1 tbMO (lower panels in A, C) together with 100 pg lacZ mRNA. Embryos were fixed at stage 15. The injected side was traced by X-gal staining (blue). 
A

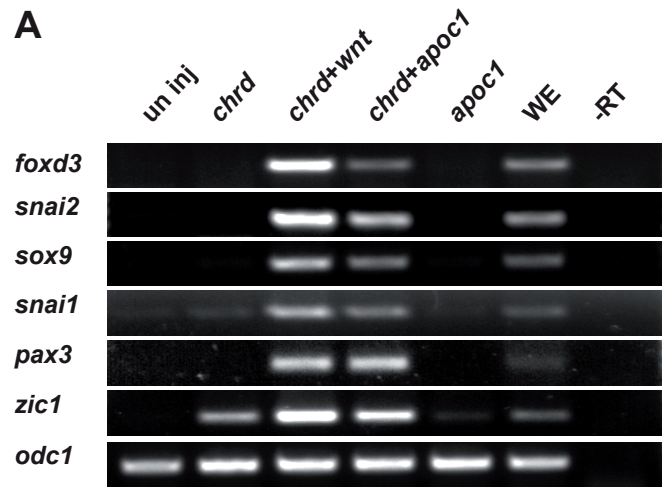

B

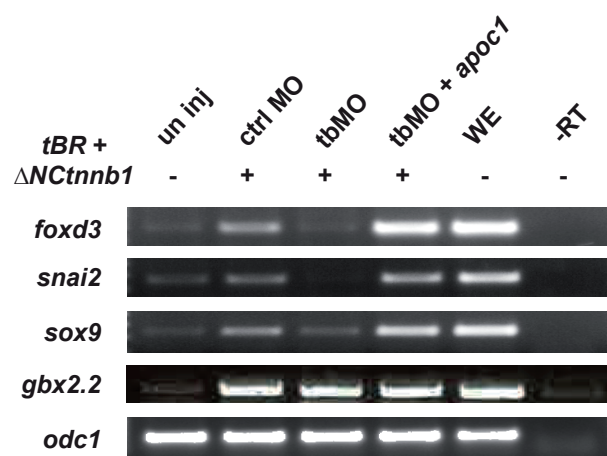

C

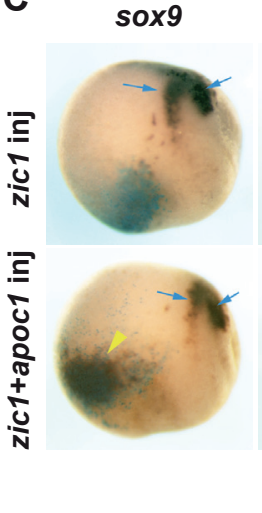

snai2

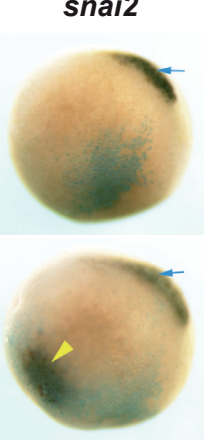

Fig. 5. Apoc1 can substitute for wnt in the animal cap neural crest induction assay. (A) $200 \mathrm{pg}$ of chrd, $200 \mathrm{pg}$ wnt $3 \mathrm{a}$ and/or $300 \mathrm{pg}$ of apoc1 was injected into two animal blastomeres of 4- or 8-cell stage embryos. Animal caps were dissected at stage 9 and cultured until stage 19. Gene expression was assayed by RT-PCR. (B) $125 \mathrm{pg}$ tBR (dominant negative form of bmpr1a) and $30 \mathrm{pg}$ aN-HA-Ctnnb1 (constitutively active form of ctnnb1) mRNA was injected in two animal blastomeres at the 4- to 8-cell stage to induce neural crest cell marker expression. In addition, 40 ng of either control (ctrl) or translation blocking Apoc1 MO (tbMO) with or without 400 pg MO-resistant apoc1 mRNA was injected to determine if Apoc1 is required for neural crest induction. Animal caps were dissected at stage 9 and cultured until stage 16. Gene expression was assayed by RT-PCR. (C) Coinjection of apoc1 and zic1 into the ventral side of the embryo induced ectopic expression of sox 9 and snai2. 50 pg of zic $1+/-500$ pg of apoc1 was injected into ventral marginal cells of 8 cell-stage embryos. The embryos were fixed at stage 14, and subjected to whole mount in situ hybridization. The blue arrows point to endogenous sox9 and snai2 expression. The yellow arrowheads show the area of ectopic sox9 and snai2 expression.

mal ectoderm, is shifted laterally (Fig. 4C; supplementary material Table S2). Furthermore, expression of the border marker $m s \times 1$, was depleted in Apoc1-depleted embryos (Fig. 4C; supplementary material Table S2). Interestingly, Msx1 morphants also exhibit an expanded sox2 expression domain, and a lateral shift of the medial $x k 81 a 1$ expression boundary (Monsoro-Burq et al., 2005).

$P a x 3$ is a border specifier and required for neural crest induction. Furthermore, it is sufficient for neural crest induction in vivo and in animal caps when ectopically expressed together with zic1 (Sato et al., 2005). It has been shown that pax3 is an immediate-early target of Wnt signaling in neural border induction (de Croze et al., 2011). We then examined pax3 expression in Apoc1 morphants and observed a dramatic downregulation in pax3 expression in the neural folds (Fig. 4C; supplementary material Table S2). This result indicates that Wnt signaling directly induces initial expression of pax3, and apoc1 maintains the expression of pax 3 in neural border. Together these results demonstrate that apoc1 is required for border formation.

\section{Apoc1 together with BMP antagonists can induce neural crest markers in a neural crest induction assay}

We have demonstrated that apoc1 is activated by Wnt/Ctnnb1 signaling both in vivo and in animal caps. The combined activity of Wnt ligands and BMP antagonists in animal caps results in a potent induction of neural crest cells (Saint-Jeannet et al., 1997). Thus, we asked if apoc1 could also induce neural crest markers with BMP antagonist in the animal cap neural crest induction assay. We injected embryos at the 4-8 cell stage with apoc1, the BMP antagonist chordin (chrd), chrd/wnt3a, and chrd/apoc1, dissected animal caps at stage 9 and cultured them until control embryos reached a late neurula stage (stages 19; Fig. 5A). As expected, RT-PCR analysis of the late neurula stage samples demonstrated that chrd alone can activate zic1 expression, but does not result in neural crest induction. However, chrd in combination with wnt3a robustly induced the neural crest specifiers foxd3, snai2, sox9, snai1, and the border specifier pax3. Expression of apoc1 alone did not activate any of the genes assayed, but in combination with chrd, apoc1 robustly induced the expression of all markers. This result suggested that apoc1 is a key mediator of Wnt/Ctnnb1 signaling in neural crest induction. To determine if apoc1 is also required for Wnt-mediated neural crest induction in animal caps, we injected a dominant negative form of bmpr1a and a constitutively active form of ctnnb1 with or without Apoc1 $\mathrm{MO}$, dissected animal caps at stage 9, and performed RT-PCR at stage 16 (Fig. 5B). Apoc1 MO coinjection strongly suppressed the NC markers foxd3, snai2 and sox9. Furthermore, the expression of these markers was restored by injecting morpholino resistant apoc1 mRNA (Fig. 5B). Taken together, apoc 1 is required for Wnt-mediated neural crest induction in animal caps. As previous study showed (Li et al., 2009), gbx2.2 was induced in the animal caps injected with dominant negative form of bmpr1a and a constitutively active form of ctnnb1, and its expression was not changed by injecting Apoc1 tbMO.

Chrd can be replaced both in vitro and in vivo with the chrdinduced gene zic1 to, in combination with e.g. pax3 or gbx2.2, induce neural crest markers (Sato et al., 2005; Li et al., 2009). As apoc1 and chrd together could induce neural crest markers in vitro, we examined if apoc1 together with zic1 could induce ectopic neural crest markers in vivo (Fig. 5C). Injection of zic1 alone in the ventral embryo could not induce sox9 or snai2, but apoc1 together with zic1 induced robust ectopic sox9 and snai2 (Fig. 5C; supplementary material Table S3).

apoc1 and gbx2.2 are both required for neural crest induction and are independently regulated by Wnt/Ctnnb1 signaling

Li et al., have suggested that $g b \times 2.2$ is the earliest target of Wnt/Ctnnb1 signaling in neural crest induction (Li et al., 2009). To determine the epistatic relationship between endogenous apoc1 and $g b x 2.2$, we analyzed apoc 1 expression in Gbx2.2 MO injected embryos and gbx2.2expression in Apoc1 MO injected embryos (Fig. $6 A-D)$. Interestingly, neither gene appeared regulated by the other 
(supplementary material Table S4), demonstrating that apoc1 and gbx2.2 are independently regulated during early Xenopus development. Fig. 5B results also indicated that $g b x 2.2$ expression in the neural crest induction assay is independent of Apoc1. In addition, the loss of the neural crest markers sox 9 and snai2 in Apoc1 or Gbx2.2 MO injected embryos could not be rescued by coinjection of $g b x 2.2$ or apoc $1 \mathrm{mRNA}$, respectively (Fig. $6 \mathrm{E}-\mathrm{H}$; supplementary material Table S4). Thus, apoc1 and gbx2.2 are both required for neural crest development, but independently regulated.

To determine whether both apoc1 and $g b \times 2.2$ are required for Wnt/Ctnnb1 mediated neural crest induction in vivo, GR-Ctnnb1 was targeted to the neural plate/neural border region and activated by DEX at an early gastrula stage. As expected, the snai2 and sox9 expression domains were both expanded anteriorly (Fig. 6I, J; supplementary material Table S4), and in some embryos, expression was even detected in the neural plate (Fig. 6I). Strong ectopic expression of both apoc1 and gbx2.2 was observed overlapping the entire area where GR-Ctnnb1 was expressed (Fig. $6 \mathrm{~K}$, L; supplementary material Table S4). In addition, $m s \times 1$ and pax3 were also found to be ectopically expressed (Fig. $6 \mathrm{M}, \mathrm{N}$; supplementary material Table S4).

We then coinjected GR-Ctnnb1 and Apoc1 tbMO or Gbx2.2 MO. The anterior expansion of snail2 was rescued in both morphants (Fig. 6 O,Q; supplementary material Table S4) demonstrating that both apoc1 and gbx2.2are important mediators of Wnt/Ctnnb1 signaling in neural crest induction in vivo. However, Apoc1 or Gbx2.2 depletion did not rescue the GR-Ctnnb1-induced ectopic expres- sion of gbx2.2 or apoc1, respectively (Fig. 6P, R; supplementary material Table S4). Taken together, the expression of apoc1 and gbx2.2 are regulated independently by Wnt/Ctnnb1 signaling, but both are required for neural crest induction in vivo.

\section{Apoc1 does not regulate regionalization of the border}

Our findings show that apoc1 is a key mediator of Wnt/Ctnnb1 signaling in border formation, but Wnt/Ctnnb1 signaling has also been reported to posteriorize the border (Patthey et al., 2008; Li et al., 2009). Therefore, to investigate whether apoc1 is sufficient for posteriorizing the neural border, apoc1 mRNA was targeted to the neural border. Unlike after expression of wnt or $g b x 2.2$ in the anterior border (Li et al., 2009), no anterior expansion of pax3 or snai2 was observed, and the preplacodal marker foxi4.1 was maintained (Fig. 7 A-C; supplementary material Table S5). Apoc1 is thus not sufficient to promote posteriorizaton of the anterior neural border.

In Gbx2.2 morphants, the posterior border is anteriorized. We therefore analyzed foxi4.1 also in Apoc1 morphants (Fig. 7 D-F). Rather than a posterior expansion, we found that foxi4. 1 was lost in the most posterior part of the preplacodal domain (Fig. 7F; supplementary material Table S5). This result shows that $g b \times 2.2$ and apoc1 have opposite effects on preplacodal development; $g b x 2.2$ inhibits, but apoc 1 is required for expression of preplacodal markers. Thus, these results argue that while apoc1 is required for mediating Wnt's border specification and neural crest induction activities, it is, unlike $g b \times 2.2$, not acting as a posteriorizing factor.
Fig. 6. Apoc1 and gbx2.2 are required for emergence of neural crest cells, but are independently regulated by Wnt signaling. (A-H) The expression of apoc1 and gbx2.2 is independently regulated, but both genes are required for neural crest induction. Apoc1 expression in Gbx2.2 (A) or control MO (B) injected embryos and gbx2.2 expression in Apoc1 (C) or control MO (D) injected embryos. The reduction of sox 9 and snai2 expression was not rescued by coinjection of 100 pg gbx2.2 mRNA in Apoc1 (E,G) or 400 pg apoc1 mRNA in Gbx2.2 (F, H) depleted embryos. $30 \mathrm{ng}$ of $\mathrm{MO}$ and $100 \mathrm{pg}$ lacZ mRNA was injected into one side of a single dorsoanimal blastomere of 8-cell stage embryos. The embryos were fixed at stage 15. (I-R) Apoc1 and gbx2.2 are both required for Wntmediated neural crest induction in vivo. $200 \mathrm{pg}$ of GR-ctnnb1 and 100 pg lacZ mRNA were injected into one side of a dorsoanimal blastomere of 8-cell stage embryos and the embryos were treated with DEX at stage 10.5. The embryos were fixed at stage 15 for whole mount in situ hybridization with snail2 (I), sox9 (J), apoc1 (K), gbx2.2 (L), msx1 (M), and pax3 (N). Ectopic expression of gbx2.2 in GR-ctnnb1 injected embryos was not reduced by coinjecting 30 ng Apoc1 tbMO (P), but the anterior expansion of snai2 was rescued $(\mathbf{O})$. Ectopic expression of apoc1 in GR-ctnnb1 injected embryos was not reduced by coinjecting $30 \mathrm{ng}$ Gbx2.2 MO (R), but the anterior expansion of snai2 was rescued (0). Blue arrowhead indicates ectopic expression. 
To further support this claim, we turned to the animal cap assay and analyzed border and neural crest markers at stage 14 (early neurula stage; Fig. 7G). As was shown in late neurula stage animal explants (Fig. 5), apoc1 (or wnt) together with chrd can induce snai2 and pax3 (Fig. 7G). The zic1 level was even in all chrd-injected animal explants, and as expected, coinjection of wnt and chrd reduced expression of the preplacodal marker six 1 and the anterior neural and placodal marker otx2 (Fig. 7G). These results confirm the posteriorizing effect of wntexpression. However, while coinjection of chrd and apoc1 induced neural crest markers, it failed to repress sox2, six 1 and otx2 (Fig. 7G). These three markers are all expressed in the anterior border or placodes. Our data thus suggests that apoc1 is required to mediate the border specification, but not the border regionalization, activity of Wnt/ Ctnnb1 signaling.

\section{Discussion}

We identified the apolipoprotein apoc1 in a screen for Wnt/ Ctnnb1-regulated genes in late blastula Xenopus tropicalisembryos. Using Xenopus laevis, we confirmed this finding and demonstrated that Apoc1-depleted tadpoles exhibited defects in the formation of neural crest- and placode-derived cell types. In vertebrates, both placodal and neural crest cells are generated from the border, albeit from distinct regions. While the anterior border produces placodal cells, the posterior border generates both neural crest (medial border) and placodal cells (lateral border). The emergence of neural crest and placodal cells are complex, multi-step processes (Meulemans and Bronner-Fraser 2004; Betancur et al., 2010), which are initiated already in the late blastula/early gastrula stage embryo at which time cells of the border are induced (Patthey and Gunhaga 2011). Our study has revealed an absolute requirement for the Wnt/Ctnnb1 signaling target apoc1 in border formation, suggesting that a Wnt signal is required in blastula stage embryos to promote border formation.

Wnt/Ctnnb1 signals are used reiteratively during neural crest development. Wnts have been clearly demonstrated to regulate antero-posterior border patterning and neural crest induction (Saint-Jeannet et al., 1997; LaBonne and Bronner-Fraser 1998). Previous studies have provided conflicting data on whether Wnt activity is required for, or actually inhibits, border formation. While one study suggested that Wnt activity needs to be repressed for border induction, and then activated for neural crest induction (Steventon and Mayor 2012), another proposed that Wnt is required to initiate a BMP-dependent neural border induction mechanism (Patthey et al., 2009). In addition, Wnt/Ctnnb1 has been shown to be required for inhibiting neural induction and promoting neural crest development (Heeg-Truesdell and LaBonne 2006). When Wnt activity was ablated using dominant negative lef1 or tcf3 constructs, or Ctnnb1 morpholinos, c-myc was repressed, the neural plate was expanded laterally and neural crest cells were not formed (Heeg-Truesdell and LaBonne 2006). Taken together with the observation that $c-m y c$ is required for border formation (Bellmeyer et al., 2003), and our findings in this study: that apoc1 functions upstream of $c$-myc in border formation and that apoc1 is a Wnt/Ctnnb1 target, argues for an early Wnt/Ctnnb1 requirement for ensuring the formation of a proper border domain. Consistent with the idea that at least the neural crest producing border cells originate from the lateral neural tube, we showed that activating Wnt

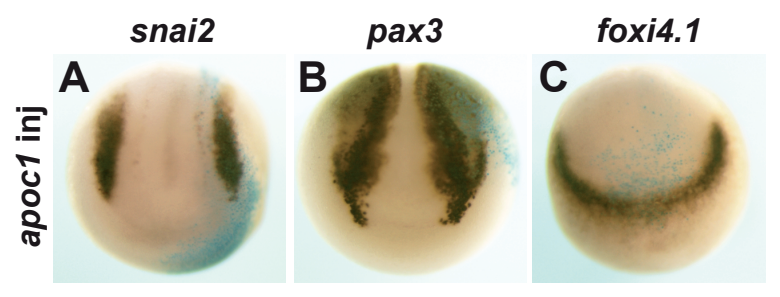

foxi4.1
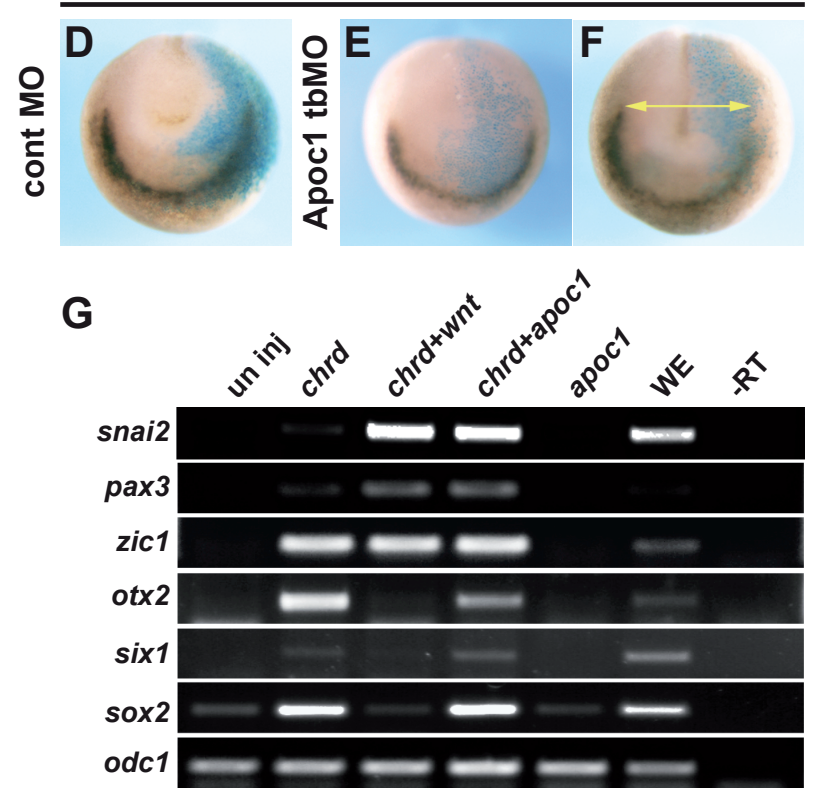

Fig. 7. Apoc1 does not posteriorize the border. (A-C) Expression of apoc1 (400 pg) is not sufficient to posteriorize the anterior neural border as demonstrated by analysis of snai2 (A), pax3 (B) and foxi4.1 (C) using in situ hybridization. One of the dorsoanimal blastomeres was injected with $400 \mathrm{pg}$ apoc1 and $100 \mathrm{pg}$ lacZ mRNAs at the 8-cell stage. (D-F) Apoc1 is required for expression of foxi4.1 in the posterior preplacodal domain. Whole mount in situ hybrydization of foxi4.1 in control MO (D) or Apoc1 tbMO (E,F) injected embryos. The yellow arrow shows the posterior limit of foxi4.1 expression on the uninjected side. $30 \mathrm{ng}$ of $\mathrm{MO}$ was injected with 100 pg of lacZ mRNA into one side of a dorsoanimal blastomere of 8-cell stage embryos and fixed at stage 15. (G) 200 pg of chrd, 200 pg wnt3a and/or $300 \mathrm{pg}$ of apoc1 was injected into two animal blastomeres at the 4- to 8-cell stage. Animal caps were dissected at stage 9 and cultured until stage 14. Gene expression was assayed by RT-PCR. Odc1 is a loading control.

signaling in the early neural plate using DEX-inducible GR-ctnnb1 results in strong ectopic expression of apoc1, gbx2.2, msx1 and pax3 in the neural plate. In fact, in some embryos, expression of snai2 was also detected in the neural domain, suggesting that a continuous Wnt/Ctnnb1 signal is sufficient to respecify some neural cells into neural crest-producing border cells.

Several studies have demonstrated that, at gastrula stages, Wnt/ Ctnnb1 is required for patterning the border into separate anterior and posterior domains. Secreted or intracellular Wnt antagonists, such as $d k k 1$ or $k c t d 15$, are required for the border to acquire anterior character. Furthermore, the anterior border expresses posterior markers upon ectopic expression of wnt or ctnnb1 and produces neural crest cells rather than placodal cells. Gbx2.2 has been shown to be a direct target of Wnt/Ctnnb1 signaling and 
to mediate its posteriorizing activity (Li et al., 2009). In Gbx2.2depleted embryos, pax3 and msx1 expression was reduced/lost and neural crest cells did not form. However, the border was formed and expressed the panplacodal marker six1. Conversely, ectopic expression of $g b x 2.2$ was sufficient for repressing six 1 expression and imposing a posterior character on the anterior border. Gbx2.2 exerts this function by crossrepressing otx2 (Steventon et al., 2012), a mechanism that also patterns the central nervous system (Nordstrom et al., 2002). In contrast, apoc1 expression in the anterior border does not repress the placodal marker foxi4.1, and conversely, foxi4.1 is not expanded posteriorly in Apoc1depleted embryos. In fact, the posterior limit of the foxi4.1 domain is shifter anteriorly, which is consistent with the observed loss of placodal fates in tadpole morphants and a requirement for apoc1 in border formation.

As pax3and $m s x 1$ is lost in Gbx2.2-depleted embryos, ithad been suggested that $g b x 2.2$ should be placed at the top of the hierarchy of genes controlling neural crest development (Li et al., 2009). However, unlike Apoc1-depleted embryos, Gbx2.2 morphants were not reported to exhibit an expanded neural plate and neural border formation defects. In fact, the border was formed but the patterning perturbed resulting in loss of neural crest fates. Given the spatiotemporal expression pattern of apoc1, first appearing in the dorsal region at the onset of gastrulation followed by expression in the border, except the most anterior part, apoc1 is likely to be the immediate Wnt-regulated genes in border formation. This prompted us to examine the epistatic relationship between apoc1 and $g b x 2.2$. We found that apoc1 and $g b x 2.2$ are independently regulated by Wnt signaling, both in the early embryo and upon targeting expression of GR-ctnnb1 to the nervous system. Based on these observations, we propose a model in which Wnt regulates border formation and border patterning by two independent genetic programs. We suggest that apoc1 mediates Wnts border formation activity and $g b x 2.2$ the anteroposterior patterning activity. Thus our study provides further insights into the genetic mechanism by which Wnt controls two distinct steps of the neural crest developmental program. Furthermore, apoc1 remains expressed after border formation, in the neural folds and migrating neural crest, suggesting that it may be required also for neural crest induction, migration or differentiation.

The cellular and molecular mechanisms by which apoc1 contributes to border formation remain to be elucidated. Apoc1 is a member of the apolipoprotein family that binds lipids to form lipoprotein such as chylomicrons, IDL, LDL, VLDL, and HDL. These lipoproteins transport lipids through the lymphatic and circulatory systems. In addition, apolipoproteins have been demonstrated to act as ligands for cell surface receptors (Li et al., 1988). There are four members of the apoc subfamily of low molecular weight apolipoproteins. The human apoc1 gene is located in the apoe/c1/ c2 gene cluster on chromosome 19 (Jong et al., 1999). Both apoc1 and apoe 4 are known to be risk factors for Alzheimer's disease (AD), possibly by controlling the cellular cholesterol homeostasis in the brain (Leduc et al., 2010), although a detailed mechanism has not been provided.

This study is one of the first to ascribe a function to apoc1 during embryonic development. The demonstration that apoc1 activity is required as early as during late blastula is interestingly since a circulatory system has yet to be established at such early stages of Xenopus development, possibly suggesting a novel molecular mechanism. In mice, both overexpression and knock out of apoc1 results in impaired memory functions (Berbee et al., 2011), but no embryonic defects have been reported. This suggests that, in mouse, there might be either redundancies, a requirement for a different apolipoprotein family member, or a different mechanism for establishing the neural border.

Several studies have shown that apoe is a physiologically relevant ligand for low-density lipopotein receptors (LDLRs) and LDLR related proteins (LRPs) (Zaiou et al., 2000). Apoc1 can also inhibit apoe-mediated $\beta$-VLVL binding to LRP (Weisgraber et al., 1990) suggesting the possibility of direct binding of apoc1 to LRPs. Therefore, we examined the possibility that apoc1 modulates Wnt signaling by using a reporter construct (TOP-flash assay; Fig. S4). Since we showed that secretion of Apoc1 protein was required for its function (Fig. 4B), it might be possible that Apoc1 cooperates with extracellular Wnt components such as Wnt ligand or the Wnt antagonist Dkk1. We, therefore, examined TOP-FLASH reporter assays using Irp6, apoc1 together with either low amount of wnt3a or dkk1 in Xenopus animal caps (Fig. S4B), however, we did not observe any activation or repression of Wnt activity when apoc1 was coexpressed (Fig. S4B). These data suggested that the molecular mechanism by which apoc1 functions in early development is not by modulating wnt signaling. Further studies are required to fully understand the molecular function of apoc1 in Xenopus embryos.

\section{Materials and Methods}

\section{Xenopus embryos and animal cap assays}

Animal care and research protocols were in accordance with institutional guidelines, and approved by the Etiska Nämnden on animal use. Xenopus laevis eggs were obtained by injecting frogs with 700 units human chorionic gonadotropin (Pregnyl $\AA^{\circledR}$, Merck Sharp \& Dohme). The eggs were fertilized using a sperm suspension. The embryos were dejellied with $1 \%$ thioglycolic acid, and cultured in 0.2x Marc's Modified Ringer's solution (MMR) at 18$20^{\circ} \mathrm{C}$. Staging was according to Nieuwkoop and Faber (Nieuwkoop and Faber 1994). Ectodermal explants (animal caps) were dissected at stage 9 in $0.2 \times$ MMR, and cultured in $0.4 \times$ MMR, $0.1 \%$ BSA until control embryos reached the desired stage. Xenopus tropicalis embryos were obtained by artificial insemination and cultured in $20 \%$ Steinberg's Solution (SS) at 23 $25^{\circ} \mathrm{C}$. Fertilized eggs were dejellied using $4 \% \mathrm{~L}-\mathrm{C}$ stein in $20 \% \mathrm{SS} \mathrm{pH} 8.0$.

\section{Microinjection and DNA constructs}

Microinjections were performed in 4\% Ficoll/0.3x MMR (Xenopus laevis) or $5 \%$ Ficoll/1x SS (Xenopus tropicalis). The maximum injection volume was $20 \mathrm{nl}$ (Xenopus laevis) or $1 \mathrm{nl}$ (Xenopus tropicalis) per embryo. The mMessage mMachine ${ }^{\circledR}$ sp6 Kit (Ambion) was used to synthesize in vitro capped mRNA. pCS2-super was generated by inserting an oligonucloetide fragment containing a polylinker sequence (EcoRI, Pacl, Sbfl, Xmal, Xhol, Ascl, Xbal) into the EcoRI/Xbal sites of pCS2. pCS2-3xFLAG was generated by inserting a oligonucleotide fragment containing 3xFLAG followed by a stop codon into the Xmal/Ascl sites of pCS2-super. To generate pCS2-apoc1, the Xenopus laevis apoc1 open reading frame (ORF) was obtained by RT-PCR from embryonic cDNA and subcloned into the Pacl/ Ascl sites of pCS2-super. pCS2-apoc1-3xFLAG was generated by PCR amplifying the apoc1 ORF without the stop codon and subcloning into the $\mathrm{Pacl} / \mathrm{Xmal}$ sites of pCS2-3xFLAG. pCS2-5'UTR-apoc1-3xFLAG was generated by subcloning a PCR fragment containing 42 base pairs of the apoc1 untranslated region directly upstream of the first methionine and the apoc1 ORF (without the stop codon) into the Pacl/Xmal sites of pCS2-3xFLAG. Wnt3a was isolated from a Xenopus tropicalis full-length clone library (Source BioScience). In addition, GR-Ctnnb1 ( $\beta$-catenin-GR), dominant negative bmpr1a (tBR), and chrd was used for making mRNA for injections. 


\section{Morpholino antisense oligos}

Morpholino antisense oligos were synthesized by Gene Tools, LLC(USA). To design the Apoc1 translation blocking morpholino (tbMO), sequences of more than 50 EST clones were compared to identify a suitable conserved sequence in the five prime untranslated region. To design the apoc1 splice blocking MO (spMO), genomic DNA was amplified by PCR with the primer for exon 1 and 3 , and several clones were sequenced, and the spMO was designed against the exon2-intron2 boundary. The sequences of the MOs are; tbMO: 5'-GGG CAC CTT CTT TAG AAA GCT CTG A-3', spMO: 5'-GTC AGG AAAAGA TAC TGTACC TTG T-3', control MO: 5'-CCT CTTACC TCA GTT ACA ATT TAT A-3'. The Gbx2.2 MO sequence has previously been reported (Li et al., 2009). To test the efficiency of the tbMO, western blots were performed with a 5'UTR apoc1-flag construct described further below.

\section{RNA isolation and RT-PCR assays}

Total RNA extraction was performed using the TRIzol RNA isolation protocol (Life Technologies). The total RNA was treated with DNAsel (Roche), and $c D N A$ was synthesized with the Maxima ${ }^{\circledR}$ First Strand cDNASynthesis Kit for RT-qPCR (Thermo Scientific). The primers used were: apoc1-fwd CGC ACT GTC TGT GAT TCT GG, apoc1-rev CAG AGC TGG ACA CCT TTT CC, nodal3.2 (Darras et al., 1997), odc1 (Heasman et al., 2000), foxd3, pax3 and zic1 (sato et al., 2005), otx2 (Mizuseki et al., 1998), sox9 and six1 (Li et al., 2009), snai1 and snai2 (LaBonne and Bronner-Fraser 1998).

\section{Blastocoel cavity fluid collection and western blot}

The blastocoel fluid was collected by inserting a thin needle connected to a PL1-100 Deluxe Pico Injector (Harvard Apparatus, Holliston, Massachusetts, USA) into the blastocoel cavity of stage 10 embryos. The blastocoel fluid from 10 embryos (approximately $0.2 \mu \mathrm{l} /$ embryo) per condition was collected. As a control, total protein from whole embryos (5-10 embryos) was extracted by homogenizing the tissue on ice in extraction buffer [Tris$\mathrm{HCl} \mathrm{pH7.5,} \mathrm{protease} \mathrm{inhibitors} \mathrm{(cOmplete,} \mathrm{Mini} \mathrm{Protease} \mathrm{Inhibitor} \mathrm{Cocktail}$ Tablets, Roche)]. Homogenates were spun at 13,000 rpm for 2 minutes. The supernatant was prepared for Western blot by mixing with an equal volume of $2 x$ reducing sample buffer and boiling. Approximately 1 embryo equivalent of protein extract was loaded on a $12 \%$ SDS-PAGE gel. The blastocoel fluid was diluted with extraction buffer, mixed with reducing sample buffer, and boiled. The entire blastocoel fluid sample was loaded on a $12 \%$ SDS-PAGE gel. The nitrocellulose membranes were blocked with $5 \%$ milk, incubated with anti-FLAG® M2 antibody (dilution 1:1125; F3165, Sigma Aldrich) overnight, and the signal was detected with SuperSignal ${ }^{\circledR}$ West Pico Chemiluminescent Substrate (Thermo Scientific). Finally, the membranes were stripped and stained with a $\alpha$-tubulin antibody (dilution 1:10000; T5168, Sigma Aldrich) as a loading control.

\section{Whole-mount in situ hybridization}

The embryos were fixed in MEMFA for 2 hours at room temperature. Digoxigenin in situ hybridization was performed as described in (Harland 1991) with some modifications. NBT/BCIP was used as substrate for alkaline phosphatase. The Xenopus laevis apoc1 probe (pBKS-apoc1) was generated by ligating an EcoRI/Xbal fragment from pCS2-apoc1 into pBluescript KS.

\section{Cycloheximide treatment}

For blocking protein synthesis, embryos injected with 200pg of GRctnnb1were treated with $100 \mu \mathrm{g} / \mathrm{ml}$ cycloheximide 30 minutes prior to the dexamethasone treatment at st11.5. Embryos were then fixed with MEMFA at st13 (apc1, gbx2.2 and pax3) or st15 (snail2), and used for whole-mount in situ hybridization.

\section{Acknowledgements}

We would like to thank to R. Mayor, J-P. Saint-Jeannet, C. LaBonne, Y. Sasai, A. Brändli, W. Knöchel, N. Itasaki, J. Smith, R. De Robertis and $M$. Asashima for providing us with plasmid constructs. This work was supported by the Wenner-Gren Foundations, Sweden [Wenner-Gren Fellows to J.M.S.] and the Ludwig Institute for Cancer Research Ltd [J.M.S].

\section{Competing interests statement}

The authors declare no competing financial interests.

\section{Author contributions}

J.M.S. and C.Y. conceived the project. J.M.S., C.Y., C.Å., S.T. designed and C.Y., C.Å., S.T. performed experiments. J.M.S., C.Y., C.A., D.W.H. analyzed data and J.M.S., C.Y., C.A. wrote the manuscript.

\section{References}

BELLMEYER A, KRASE J, LINDGREN J, LABONNE C (2003). The protooncogene c-myc is an essential regulator of neural crest formation in Xenopus. Dev Cell 4: 827-839.

BERBEE J F, VANMIERLO T, ABILDAYEVA K, BLOKLAND A, JANSEN P J, LUTJOHANN D, GAUTIER T, SIJBRANDS E, PRICKAERTS J, HADFOUNE $M$ et al., (2011). et al.Apolipoprotein $\mathrm{Cl}$ knock-out mice display impaired memory functions. J Alzheimers Dis 23: 737-747.

BETANCUR P, BRONNER-FRASER M, SAUKA-SPENGLER T (2010). Assembling neural crest regulatory circuits into a gene regulatory network. Annu Rev Cell Dev Biol 26: 581-603.

BOLANOS-GARCIA V M, MIGUEL R N (2003). On the structure and function of apolipoproteins: more than a family of lipid-binding proteins. Prog Biophys $\mathrm{Mol}$ Biol 83: 47-68.

DARRASS, MARIKAWAY, ELINSONRP, LEMAIREP (1997). Animal and vegetal pole cells of early Xenopus embryos respond differently to maternal dorsal determinants: implications for the patterning of the organiser. Development 124: 4275-4286.

DE CROZE N, MACZKOWIAK F, MONSORO-BURQ A H. (2011). Reiterative AP2a activity controls sequential steps in the neural crest gene regulatory network. Proc Natl Acad Sci USA 108: 155-160.

DUBAND J L (2010). Diversity in the molecular and cellular strategies of epithelium-tomesenchyme transitions: Insights from the neural crest. Cell Adh Migr 4: 458-482.

GOHIN M, BOBE J, CHESNEL F (2010). Comparative transcriptomic analysis of follicle-enclosed oocyte maturational and developmental competence acquisition in two non-mammalian vertebrates. BMC Genomics 11: 18

HARLAND R M (1991). In situ hybridization: an improved whole-mount method for Xenopus embryos. Methods Cell Biol 36: 685-695.

HEASMAN J, KOFRON M, WYLIE C (2000). Beta-catenin signaling activity dissected in the early Xenopus embryo: a novel antisense approach. Dev Biol222: 124-134.

HEEG-TRUESDELL E, LABONNE C (2006). Neural induction in Xenopus requires inhibition of Wnt-beta-catenin signaling. Dev Biol 298: 71-86.

HONG C S, PARK B Y, SAINT-JEANNET J P (2008). Fgf8a induces neural crest indirectly through the activation of Wnt8 in the paraxial mesoderm. Development 135: 3903-3910.

JONG M C, HOFKER M H, HAVEKES L M (1999). Role of ApoCs in lipoprotein metabolism: functional differences between ApoC1, ApoC2, and ApoC3. Arterioscler Thromb Vasc Biol 19: 472-484

LABONNE C, BRONNER-FRASER M (1998). Neural crest induction in Xenopus: evidence for a two-signal model. Development 125: 2403-2414.

LEDUC V, JASMIN-BELANGER S, POIRIER J (2010). APOE and cholesterol homeostasis in Alzheimer's disease. Trends Mol Med 16 469-477.

LI B, KURIYAMA S, MORENO M, MAYOR R (2009). The posteriorizing gene Gbx2 is a direct target of Wnt signalling and the earliest factor in neural crest induction. Development 136: 3267-3278.

LI W H, TANIMURA M, LUO C C, DATTA S, CHAN L (1988). The apolipoprotein multigene family: biosynthesis, structure, structure-function relationships, and evolution. J Lipid Res 29: 245-271.

LIGHTW, VERNONAE, LASORELLAA, IAVARONEA, LABONNEC (2005). Xenopus Id3 is required downstream of Myc for the formation of multipotent neural crest progenitor cells. Development 132: 1831-1841.

MEULEMANS D, BRONNER-FRASER M (2004). Gene-regulatory interactions in neural crest evolution and development. Dev Cell 7: 291-299.

MILET C, MONSORO-BURQ A H (2012). Neural crest induction at the neural plate border in vertebrates. Dev Biol 366: 22-33. 
MIZUSEKI K, KISHI M, MATSUI M, NAKANISHI S, SASAI Y (1998). Xenopus Zicrelated-1 and Sox-2, two factors induced by chordin, have distinct activities in the initiation of neural induction. Development 125: 579-587.

MONSORO-BURQ A H, WANG E, HARLAND R (2005). Msx1 and Pax3 cooperate to mediate FGF8 and WNT signals during Xenopus neural crest induction. Dev Cell 8: 167-178

NIEUWKOOP P, FABER J (1994). Normal Table of Xenopus laevis (Daudin): A systematical and chronological survey of the development from the fertilized egg till the end of metamorphosis. New York \& London: Garland Publishing, Inc.

NORDSTRO U, JESSELL T M, EDLUND T (2002). Progressive induction of caudal neural character by graded Wnt signaling. Nat Neurosci 5: 525-532.

PATTHEY C, EDLUND T, GUNHAGA L (2009). Wnt-regulated temporal control of BMP exposure directs the choice between neural plate border and epidermal fate. Development 136: 73-83.

PATTHEY C, GUNHAGA L (2011). Specification and regionalisation of the neural plate border. Eur J Neurosci 34: 1516-1528

PATTHEY C, GUNHAGA L, EDLUND T (2008). Early development of the central and peripheral nervous systems is coordinated by Wnt and BMP signals. PLoS One 3: e1625.

PEGORARO C, MONSORO-BURQAH (2013). Signaling and transcriptional regulation in neural crest specification and migration: lessons from Xenopus embryos. Wiley Interdisciplinary Reviews: Dev. Biol. 2: 247-259.
SAINT-JEANNET JP, HEX, VARMUSHE, DAWIDIB (1997). Regulation of dorsal fate in the neuraxis by Wnt-1 and Wnt-3a. Proc Natl Acad Sci USA 94: 13713-13718.

SATO T, SASAI N, SASAI Y (2005). Neural crest determination by co-activation of Pax3 and Zic1 genes in Xenopus ectoderm. Development 132: 2355-2363.

SAUKA-SPENGLER T, BRONNER-FRASER M (2008). A gene regulatory network orchestrates neural crest formation. Nat Rev Mol Cell Biol 9: 557-568.

STEVENTON B, MAYOR R (2012). Early neural crest induction requires an initial inhibition of Wnt signals. Dev Biol 365: 196-207.

STEVENTON B, MAYOR R, STREIT A (2012). Mutual repression between Gbx2 and Otx2 in sensory placodes reveals a general mechanism for ectodermal patterning. Dev Biol 367: 55-65.

THEVENEAU E, MAYOR R (2012). Neural crest delamination and migration: from epithelium-to-mesenchyme transition to collective cell migration. Dev Biol 366: 34-54.

WEISGRABER K H, MAHLEY R W, KOWAL R C, HERZ J, GOLDSTEIN J L, BROWN M S (1990). Apolipoprotein C-I modulates the interaction of apolipoprotein $E$ with beta-migrating very low density lipoproteins (beta-VLDL) and inhibits binding of beta-VLDL to low density lipoprotein receptor-related protein. J Biol Chem 265 22453-22459.

ZAIOU M, ARNOLD K S, NEWHOUSE Y M, INNERARITY T L, WEISGRABER K H, SEGALL M L, PHILLIPS M C, LUND-KATZ S (2000). Apolipoprotein E;-low density lipoprotein receptor interaction. Influences of basic residue and amphipathic alpha-helix organization in the ligand. J Lipid Res 41: 1087-1095. 


\section{Further Related Reading, published previously in the Int. J. Dev. Biol.}

Bone morphogenetic protein 4 promotes craniofacial neural crest induction from human pluripotent stem cells Sumiyo Mimura, Mika Suga, Kaori Okada, Masaki Kinehara, Hiroki Nikawa and Miho K. Furue Int. J. Dev. Biol. (2016) 60: 21-28

http://dx.doi.org/10.1387/ijdb.160040mk

Xnr3 affects brain patterning via cell migration in the neural-epidermal tissue boundary during early Xenopus embryogenesis Mariko Morita, Satoshi Yamashita, Shinya Matsukawa, Yoshikazu Haramoto, Shuji Takahashi, Makoto Asashima and Tatsuo Michiue Int. J. Dev. Biol. (2013) 57: 779-786

http://dx.doi.org/10.1387/ijdb.130161tm

\section{Essential role of AWP1 in neural crest specification in Xenopus}

Jeong-Han Seo, Dong-Seok Park, Mina Hong, Eun-Ju Chang and Sun-Cheol Choi

Int. J. Dev. Biol. (2013) 57: 829-836

http://dx.doi.org/10.1387/ijdb.130109sc

KDEL tagging: a method for generating dominant-negative inhibitors of the secretion of TGF-beta superfamily proteins Shinya Matsukawa, Yuki Moriyama, Tadayoshi Hayata, Haruka Sasaki, Yuzuru Ito, Makoto Asashima and Hiroki Kuroda

Int. J. Dev. Biol. (2012) 56: 351-356

http://dx.doi.org/10.1387/ijdb.123514sm

The Wnt signaling mediator tcf1 is required for expression of foxd 3 during Xenopus gastrulation

Sylvie Janssens, Olaf Van Den Broek, lan R. Davenport, Robbert C. Akkers, Fei Liu, Gert Jan C. Veenstra, Stefan Hoppler, Kris Vleminckx and Olivier Destrée

Int. J. Dev. Biol. (2013) 57: 49-54

http://dx.doi.org/10.1387/ijdb.120191kv

Identification and characterization of Xenopus kctd15, an ectodermal gene repressed by the FGF pathway

Chika Takahashi, Toshiyasu Suzuki, Eisuke Nishida and Morioh Kusakabe

Int. J. Dev. Biol. (2012) 56: 393-402

http://dx.doi.org/10.1387/ijdb.113333ct

Xler2 is required for convergent extension movements during Xenopus development Sung-Kook Hong, Kosuke Tanegashima and Igor B. Dawid Int. J. Dev. Biol. (2011) 55: 917-921

http://dx.doi.org/10.1387/ijdb.113288sh

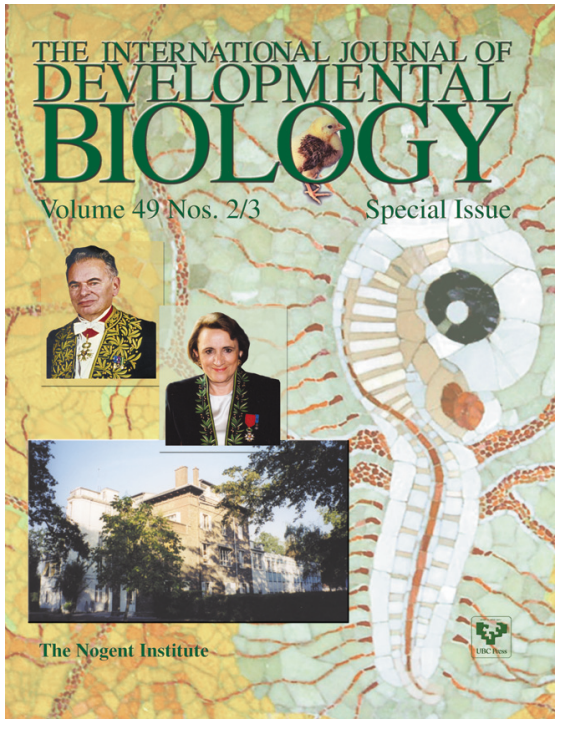

5 yr ISI Impact Factor $(2013)=2.879$
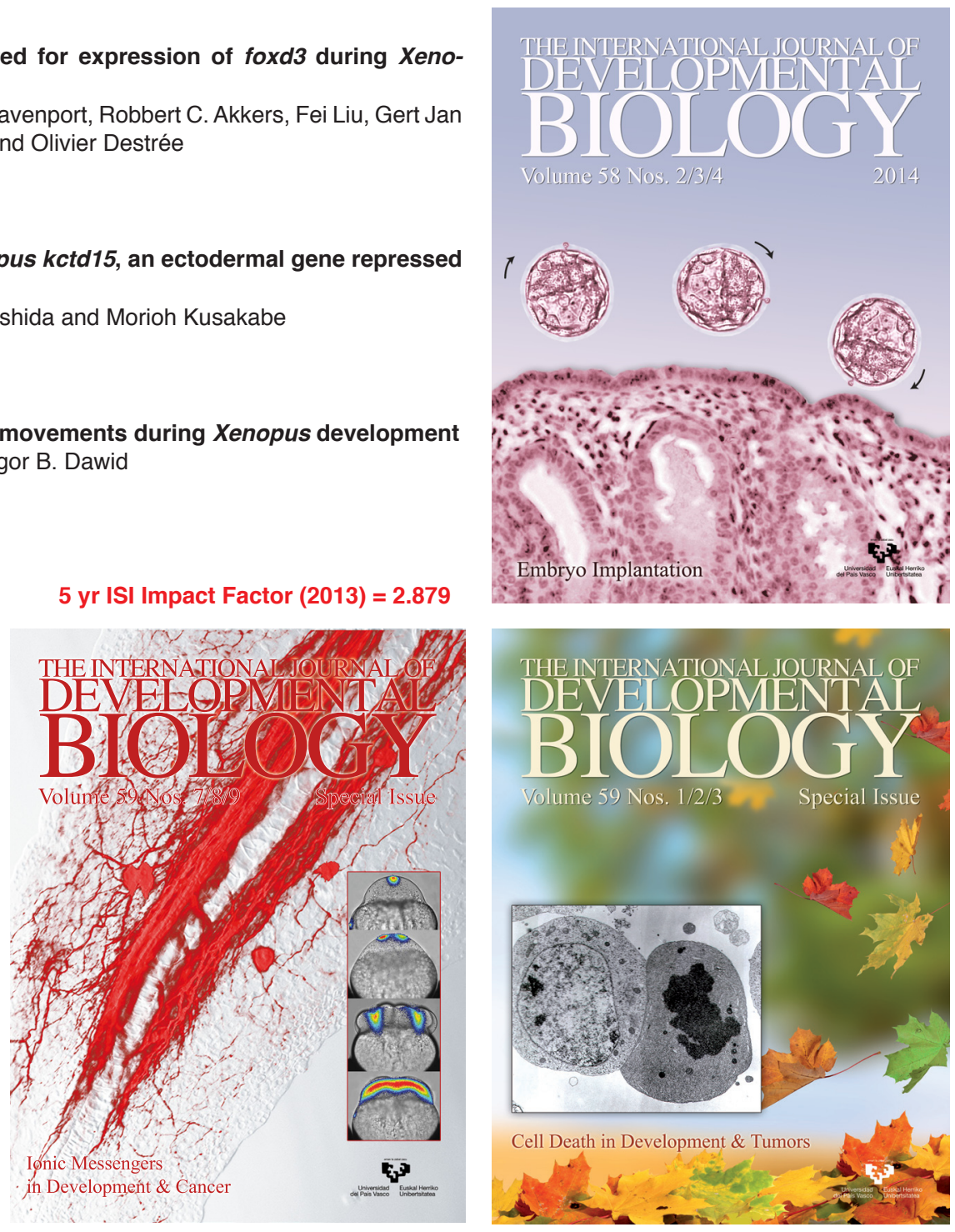